
ZS Research Square
Preprints are preliminary reports that have not undergone peer review.
They should not be considered conclusive, used to inform clinical practice, or referenced by the media as validated information.

\title{
Development and External Validation of a Nomogram for Neurosyphilis Diagnosis Among Non-HIV Patients: A Cross-sectional Study
}

\author{
Wenjing Ge \\ Sichuan University \\ Yang Zhang \\ Sichuan University \\ Chao Peng \\ Sichuan University \\ Dongdong Li \\ Sichuan University \\ Lijie Gao \\ Sichuan University \\ Jiajia Bao \\ Sichuan University \\ Changling Li \\ Sichuan University \\ Ning Chen \\ Sichuan University \\ Dong Zhou \\ Sichuan University \\ Li He ( $\square$ heli2003new@126.com ) \\ Sichuan University
}

\section{Research Article}

Keywords: Neurosyphilis, Diagnostic model, HIV-negative patient, Serum TRUST, Psychiatric symptoms

Posted Date: August 18th, 2021

DOI: https://doi.org/10.21203/rs.3.rs-778055/v1

License: @ (i) This work is licensed under a Creative Commons Attribution 4.0 International License. Read Full License

Version of Record: A version of this preprint was published at BMC Neurology on November 18th, 2021. See the published version at https://doi.org/10.1186/s12883-021-02454-8. 


\section{Abstract}

Background:

The diagnosis of neurosyphilis (NS) is challenging due to the requirement of a lumbar puncture and cerebrospinal fluid (CSF) tests.

\section{Methods:}

This cross-sectional study constructed diagnostic models for diagnosing NS with data gathered from the Western China Hospital of Sichuan University between September 2015 and April 2021 and Shangjin hospital between September 2019 and April 2021, which formed the development and validation cohorts, respectively. A logistic analysis was performed to predict reactive neurosyphilis diagnosis and was presented as a nomogram. Clinical usefulness of the diagnostic models was assessed using a receiver operating characteristic (ROC) curve analysis, and Harrell $\mathrm{C}$ index was used for discrimination and calibration plots for accuracy, adopting bootstrap resampling 500 times.

Results:

Overall, 149 and 67 patients were included in development and validation cohorts, respectively. Of those, 131 were diagnosed as having reactive NS with positive results in CSF treponemal and non-treponemal tests. In the development cohort, male sex, psychiatric symptoms, and serum toluidine red unheated serum test were selected as diagnostic indicators. The model reached $80 \%$ specificity, $79 \%$ sensitivity, and $0 \cdot 85$ area under the curves (AUC) (95\% $\mathrm{Cl}$, $0 \cdot 76$ 0.91). In the validation cohorts, the Harrell C index for the diagnostic possibility of reactive NS was $0 \cdot 71$.

\section{Conclusion:}

A convenient model using gender, presence of psychiatric symptoms and serum TRUST titre was developed and validated to indicate diagnostic results in patients suspected of NS. Checking model value of factors on nomogram is a feasible way to assist clinicians and primary health servers in updating patients' medical chart and making quantitatively informed decision on NS diagnosis.

\section{Trial registration:}

This research was retrospectively registered in Ethics committee on biomedical research, West China Hospital of Sichuan University.

\section{Research In Context \\ Evidence before this study}

Through the currently followed medical reporting system used in China, neurosyphilis may be misdiagnosed or undiagnosed in many cases. Therefore, diagnostic models are required. However, only few indicators of neurosyphilis diagnosis in the post antibiotic era have been reported. Hitherto, the association of neurosyphilis with male sex, symptoms, titre of TRUST, and titre of TPPA have been reported in several studies since 2000 , with the main risk factor being men who have sex with men in Britain and co-infection of human immunodeficiency virus in America.

\section{Added value of this study}

In this study, we found that Chinese male patients without human immunodeficiency virus (HIV) were relatively more diagnosed with neurosyphilis than female patients. This implicated that physiological sex difference contributes to sensitivity of infection to men, except for immunosuppression of HIV. We also found that numerical values of serum titres, instead of binary positive or negative results, can provide more information to clinicians and patients, enabling them to make evidence-based decisions. Although creatine kinase was linked to neurosyphilis diagnosis, the result of the single variable analysis from our data did not support this correlation even in the subgroup analysis. Moreover, psychiatric symptoms can be used as an indicator of neurosyphilis.

From the neurosyphilis diagnosis perspective, the model reached $80 \%$ specificity, $79 \%$ sensitivity, and 0.85 area under the curves (95\% confidence interval, 0.76-0.91). In the validation cohorts, the Harrell $C$ index for the diagnostic possibility of reactive neurosyphilis was $0 \cdot 71$.

Finally, this is the first report that summarizes the neuropsychiatric characteristics of neurosyphilis in West China in recent years.

\section{Implications of all the available evidence}

We utilized data from evidence before this study to develop and validate a diagnostic model, which was visualized in a nomogram by multiple logistic regression. We believe that this model could be especially useful and economical in under-developed areas for diagnosing neurosyphilis.

\section{Introduction}

Neurosyphilis (NS) is one of the most feared complications of syphilis, ${ }^{1}$ and the dissemination of the pathogenic bacterium of NS, Treponema pallidum, subspecies pallidum, to the cerebrospinal fluid (CSF) and meninges can occur at any stage of the infection. ${ }^{1}$ Importantly, the injury to brain tissues caused by TP invasion is irreversible. ${ }^{1}$ Trend results from syphilis notification data of the 25 countries with comprehensive surveillance systems showed an increase, especially in Europe, of up to $70 \%$ since $2000 .{ }^{2,3}$ However, the proportion of NS among patients with syphilis is undetermined due to diagnostic limitations and 
requirement of skilful doctors to perform lumbar puncture and lab operators for special tests. ${ }^{4}$ Further, necessary resources are usually not available to primary community healthcare centres of urban districts or common hospitals in smaller areas. ${ }^{5}$

Prior to the advent of antibiotics, the typical symptoms of NS, such as Argyll Robertson pupils, were used to diagnose NS. ${ }^{1}$ However, access to antibiotics has greatly increased and affected the disease process and manifestation of NS. ${ }^{6}$ Whether only signs and symptoms can be used for NS identification is controversial. In the recent years, headache and blurred vision are reported as supportive factors for NS diagnosis, ${ }^{7,8}$ while other reports suggest various clinical manifestations of NS with a lack of specificity. ${ }^{9}$ Furthermore, most of the descriptions of NS symptoms are derived from reports on American cohorts co-infected with $\mathrm{HIV}^{8,10}$ and there is a lack information on non-HIV patients with NS, which constitute the majority of patients with NS in Europe and Asia. 9,11

The laboratory diagnosis of NS was putatively based on positive results from serum and CSF serologic tests, as well as elevations in CSF white-cell count and protein levels. ${ }^{12,13}$ In 2015, an American guideline from the Centers for Disease Control and Prevention (CDC), U.S. department of health and human services, suggested the use of a decision tree for NS diagnosis, which required a positive or reactive definition of non-specific, specific, or alternative tests in CSF for patients suspected with NS. ${ }^{12}$ A European guideline of the European Academy of Dermatology and Venerology recommends the cut-off value of CSF treponemal tests in patients co-infected with HIV. ${ }^{13}$ Recent studies estimating serologic cut-off values have found that the accuracy of NS diagnosis depends on the choice of controls with various clinical characteristics. ${ }^{14}$ Combined the usage of diagnostic tests for NS needs further validation in the post-antibiotics era.

Variables in continuous form, instead of the traditional binary form as positive or negative report results referring to a certain threshold, can be utilised completely in the current analysis strategies for evidence-based medicine. ${ }^{15}$ By combining continuous variables with clinical parameters and presenting them as a visual graph, a nomogram makes the results of a diagnostic model simpler to use. Furthermore, this single numerical estimate of the probability of an event facilitates the evaluation of patients with NS, especially in poor areas that lack expert operators and resources to perform time-consuming tests. ${ }^{16}$ Serum tests and CSF assessments have been extensively discussed as objective indicators of NS, supporting the diagnosis; however, their usefulness remains inconclusive. 8,17 The present study was designed to verify and explore the association between these factors for diagnostic confirmation using retrospective patient data. Using the patients' clinical and laboratory characteristics, we developed a feasible diagnostic nomogram to assess the possibility of NS in an HIV-negative population with an unknown syphilis duration, and further validated a score and nomogram.

\section{Methods}

This study included consecutive patients, who presented with positive results of serum treponemal (Treponema pallidum chemiluminescence assay [TP CLIA] or Treponema pallidum particle agglutination assay [TPPA]) and a non-treponemal serological test (toluidine red unheated serum test [TRUST]) at the West China Hospital, Medical College of Sichuan University, from September 2015 to April 2021 and at Shangjin hospital between September 2019 and April 2021. Patients met one of the following criteria: presence of neurological or ophthalmological symptoms or signs (such as headache, photophobia, blurred version, confusion, sleep disorders, vertigo, hearing loss, version loss, confusion, lethargy, memory change, progressive dementia, psychiatric symptoms, personality change, numbness, fatigue or pain in limbs and trunk, seizure, tremor) and no symptom or syphilis of unknown duration, or failure of antibiotic treatment (titre of serum non-treponemal test failing to decrease by 4 fold or unable to serorevert following antibiotic treatment).

All methods were carried out in accordance with Transparent Reporting of a multivariable prediction model for Individual Prognosis or Diagnosis (TRIPOD) guidelines and regulations.

The ethics committee on biomedical research, West China Hospital of Sichuan University approved the study and waived informed consent from all subjects. The committee's reference number was 1163 in 2020 approval.

\section{Diagnostic criteria}

Subjects who were enrolled at West Hospital of Sichuan University (nヌ270) formed the development cohort and those enrolled at Shangjin Hospital ( $\mathrm{n}=95$ ) formed the validation cohort. Overall, 135 patients fulfilled all the following inclusion criteria: (1) positive serum TPPA and TP CLIA results and (2) positive CSF TRUST and CSF TPPA tests. Patients were not enrolled if they met the following exclusion criteria: (1) HIV diagnosis ( $\mathrm{n}=38$ ); (2) severe psychiatric diseases history $(n=13)$; $(3)$ death due to other emergent or severe disease $(n=11)$. We applied a strict diagnostic criterion with a combination of two laboratory methods to ensure diagnostic specificity among the suspected participants. Thus, patients with double positive results for both the CSF TRUST and CSF TPPA were assigned to the confirmed reactive NS group, and the others were assigned to the control group. In addition, 21 patients had lost their results or only had one result of CSF treponemal or non-treponemal tests, and 23 refused further tests decided by patient or their relatives.

\section{Laboratory methods}

Serum samples were collected within four days of the lumbar puncture. ${ }^{8}$ A serum non-treponemal test, TRUST (Rongsheng, Shanghai, China) and treponemal test, TPPA (Fujirebio, Tokyo, Japan) or chemiluminescent immunoassay (CLIA囚Fujirebio, Tokyo, Japan), and Lumipulse G TP-N syphilis (Fujirebio, Tokyo, Japan) were performed.

\section{Statistics}


Associations between categorical variables were assessed using a chi-squared test or Fisher's exact test. Associations between continuous variables and categorical variables were assessed using a Mann-Whitney $U$ test. Diagnostic factors were analysed and selected using univariable and multivariable regression in a stepwise manner, for confirmation of reactive NS and the other group including non-reactive and not NS- as binary classification out of clinical consideration. A two-tailed $P$ value $>0.05$ was used for the removal of variables. CSF items from clinical guidelines were assessed for possible additional

effects, among which the collinearity was tested. Boot strapping was resampled 500 times to obtain a $95 \%$ confidence interval and quantify the effects of diagnostic indicator selection strategies on the model development. Performance measures included the average area under the ROC curve, sensitivity, specificity, a calibration plot, and the Harrell C index. All analyses were weighted according to the analytical guidelines. P-values $<0 \cdot 05$ were considered statistically significant. R software (version 3.3.1; http://www.R-project.org) was used for the analysis.

\section{Results}

Table 1

Demographic and clinical features of the participants 


\begin{tabular}{|c|c|c|c|c|c|c|}
\hline & Development Cohort & & & Validation Cohort & & \\
\hline Diagnosis & Not or non-reactive NS & Reactive NS & $P$ value & Not or non-reactive NS & Reactive NS & $P$ value \\
\hline $\mathrm{N}$ & 44 & 104 & & 40 & 27 & \\
\hline Sex, n (\%) & & & 0.219 & & & 0.432 \\
\hline Male & $33(75 \cdot 0)$ & $87(83 \cdot 7)$ & & $26(65 \cdot 0)$ & $20(74 \cdot 1)$ & \\
\hline Female & $11(25 \cdot 0)$ & $17(16 \cdot 3)$ & & $14(35 \cdot 0)$ & $7(25 \cdot 9)$ & \\
\hline Age, mean (sd), year & $46 \cdot 4(13 \cdot 7)$ & $47 \cdot 3(11 \cdot 8)$ & 0.694 & $48 \cdot 75(13 \cdot 40)$ & $52 \cdot 96(16 \cdot 32)$ & $0 \cdot 252$ \\
\hline Height, mean (sd), cm & $164 \cdot 3(7 \cdot 8)$ & $166 \cdot 5(7 \cdot 7)$ & $0 \cdot 208$ & $165 \cdot 50(8 \cdot 46)$ & $166 \cdot 63(7 \cdot 98)$ & 0.585 \\
\hline Weight, mean (sd), kg & $61 \cdot 4(9 \cdot 6)$ & $62 \cdot 9(11 \cdot 4)$ & 0.542 & $71 \cdot 13(20 \cdot 89)$ & $68.92(12.59)$ & 0.642 \\
\hline Education level, n (\%) & & & $0 \cdot 110$ & & & $0 \cdot 197$ \\
\hline High school or beyond & $19(44 \cdot 2)$ & $30(30 \cdot 3)$ & & $13(32 \cdot 5)$ & $13(48 \cdot 1)$ & \\
\hline Beyond high school & $24(55 \cdot 8)$ & $69(69 \cdot 7)$ & & $27(67 \cdot 5)$ & $14(51 \cdot 9)$ & \\
\hline Address, n (\%) & & & 0.088 & & & $0 \cdot 202$ \\
\hline City & $24(55 \cdot 8)$ & $33(33 \cdot 0)$ & & $9(22 \cdot 5)$ & $5(18 \cdot 5)$ & \\
\hline Urban-rural fringe area & $5(11 \cdot 6)$ & $14(14 \cdot 0)$ & & $6(15 \cdot 0)$ & $5(18 \cdot 5)$ & \\
\hline Village and town & $14(32 \cdot 6)$ & $47(47 \cdot 0)$ & & $18(45 \cdot 0)$ & $17(63 \cdot 0)$ & \\
\hline Other province of China & $0(0 \cdot 0)$ & $4(4 \cdot 0)$ & & $6(15 \cdot 0)$ & $0(0 \cdot 0)$ & \\
\hline Aboard & $0(0 \cdot 0)$ & $2(2 \cdot 0)$ & & $1(2 \cdot 5)$ & $0(0 \cdot 0)$ & \\
\hline Treatment before, $\mathrm{n}(\%)$ & $15(43 \cdot 9)$ & $28(27 \cdot 7)$ & 0.207 & $22(55 \cdot 0)$ & $12(44 \cdot 4)$ & 0.347 \\
\hline \multicolumn{7}{|l|}{ Clinical symptoms, n (\%) } \\
\hline No symptom & $6(13 \cdot 6)$ & $8(7 \cdot 7)$ & 0.259 & $5(12 \cdot 5)$ & $3(11 \cdot 1)$ & 0.863 \\
\hline Psychiatric symptom & $9(20 \cdot 5)$ & $52(50 \cdot 0)$ & $<0.001$ & $8(20 \cdot 0)$ & $7(25 \cdot 9)$ & 0.568 \\
\hline Memory change & $9(20 \cdot 5)$ & $40(38 \cdot 5)$ & 0.033 & $7(17 \cdot 5)$ & $5(18 \cdot 5)$ & 0.915 \\
\hline Sleep difficulty & $5(11 \cdot 4)$ & $23(22 \cdot 1)$ & $0 \cdot 127$ & $1(2 \cdot 5)$ & $1(3 \cdot 7)$ & 0.776 \\
\hline Photophobia & $6(13 \cdot 6)$ & $8(7 \cdot 7)$ & 0.259 & $2(5 \cdot 0)$ & $1(3 \cdot 7)$ & $0 \cdot 801$ \\
\hline Blurred Version & $4(9 \cdot 1)$ & $6(5 \cdot 8)$ & $0 \cdot 462$ & $3(7 \cdot 5)$ & $1(3 \cdot 7)$ & 0.520 \\
\hline TP CLIA, mean (sd) & - & - & - & $57 \cdot 3(42 \cdot 0)$ & $93 \cdot 3(49 \cdot 9)$ & 0.002 \\
\hline $1<X<100, n(\%)$ & $7(16 \cdot 3)$ & $1(1 \cdot 0)$ & & $24(60 \cdot 0)$ & $7(25 \cdot 9)$ & \\
\hline$x>100, n(\%)$ & $36(83 \cdot 7)$ & $99(99 \cdot 0)$ & & $16(40 \cdot 0)$ & $20(74 \cdot 1)$ & \\
\hline Creatine kinase, (sd), U/L & $106 \cdot 5(137 \cdot 3)$ & $196 \cdot 5(373 \cdot 0)$ & $0 \cdot 132$ & - & - & - \\
\hline Serum TRUST, n (\%) & & & $<0.001$ & & & 0.008 \\
\hline Negative & $9(22 \cdot 5)$ & $3(3 \cdot 0)$ & & $15(37 \cdot 5)$ & $1(4 \cdot 0)$ & \\
\hline 1 & $14(35 \cdot 0)$ & $9(9 \cdot 0)$ & & $19(47 \cdot 5)$ & $11(44 \cdot 0)$ & \\
\hline 8 & $5(12 \cdot 5)$ & $7(7 \cdot 0)$ & & $4(10 \cdot 0)$ & $5(20 \cdot 0)$ & \\
\hline 16 & $6(15 \cdot 0)$ & $18(18 \cdot 0)$ & & $1(2 \cdot 5)$ & $3(12 \cdot 0)$ & \\
\hline 32 & $4(10 \cdot 0)$ & $30(30 \cdot 0)$ & & $0(0 \cdot 0)$ & $1(4 \cdot 0)$ & \\
\hline$\geq 64$ & $2(5 \cdot 0)$ & $33(33 \cdot 0)$ & & $1(2 \cdot 5)$ & $4(16 \cdot 0)$ & \\
\hline Serum TPPA > 1:320, $\mathrm{n}(\%)$ & $36(81 \cdot 8)$ & $96(92 \cdot 3)$ & 0.060 & $35(87 \cdot 5)$ & $25(96 \cdot 2)$ & $0 \cdot 130$ \\
\hline
\end{tabular}

\section{Demographic and clinical features}

The demographic and clinical features of the 215 clinically suspected patients with NS with complete information of treponemal, non-treponemal serum, and CSF examination are summarised in Table 1 . Among the 148 patients in the development cohort, $83 \cdot 7 \%$ were male; 14 (21.3\%) were non-symptomatic patients 
and required a CSF test. Further, 105 patients (70.9\%) did not receive any prior antibiotic treatment. The most common symptoms of NS were psychiatric symptoms ( $50.0 \%$ in the development and $25.9 \%$ in the validation cohort). Other symptoms such as sleep disorders, photophobia, and blurred version were also observed (Supplement 1).

\section{Table 2}

\section{Univariate logistic regression analysis of each indicator and diagnostic in the development cohort}

\begin{tabular}{|c|c|c|c|}
\hline \multicolumn{4}{|c|}{ Univariable Analysis } \\
\hline Characteristic & Odds Ratio & $95 \% \mathrm{Cl}$ & $P$ value \\
\hline Male & $1 \cdot 71$ & $(0 \cdot 72,4 \cdot 02)$ & 0.222 \\
\hline Age & $1 \cdot 01$ & $(0.98,1.04)$ & 0.691 \\
\hline Treatment before & 0.53 & $(0 \cdot 25,1 \cdot 11)$ & 0.094 \\
\hline Psychiatric symptoms & $3 \cdot 89$ & $(1 \cdot 70,8 \cdot 89)$ & 0.001 \\
\hline No symptom & 0.53 & $(0 \cdot 17,1 \cdot 62)$ & $0 \cdot 263$ \\
\hline Serum TPPA > 1:320 & $2 \cdot 67$ & $(0.93,7.64)$ & 0.068 \\
\hline \multicolumn{4}{|l|}{ Serum TRUST } \\
\hline Negative & Ref & & \\
\hline 1 & 1.93 & $(0 \cdot 41,9 \cdot 10)$ & 0.407 \\
\hline 8 & $4 \cdot 20$ & $(0 \cdot 74,23 \cdot 91)$ & $0 \cdot 106$ \\
\hline 16 & $9 \cdot 00$ & $(1 \cdot 82,44 \cdot 59)$ & 0.007 \\
\hline 32 & $22 \cdot 50$ & $(4 \cdot 23,119 \cdot 77)$ & 0.000 \\
\hline 64 & $49 \cdot 50$ & $(7 \cdot 15,342 \cdot 77)$ & $<0.0001$ \\
\hline Serum creatine kinase & $1 \cdot 00$ & $(1 \cdot 00,1 \cdot 01)$ & $0 \cdot 109$ \\
\hline Serum IgG & $1 \cdot 03$ & $(0 \cdot 90,1 \cdot 18)$ & 0.687 \\
\hline Serum albumin & 0.95 & $(0.88,1 \cdot 04)$ & $0 \cdot 284$ \\
\hline CSF Protein & $6 \cdot 51$ & $(1 \cdot 77,23 \cdot 96)$ & 0.005 \\
\hline$<=0 \cdot 45$ & Ref & & \\
\hline$>0 \cdot 45$ & $4 \cdot 80$ & $(2 \cdot 22,10 \cdot 36)$ & $<0.0001$ \\
\hline CSF Glucose & 0.66 & $(0.39,1.09)$ & $0 \cdot 105$ \\
\hline CSF Nucleated cells & $1 \cdot 00$ & $(0.99,1.01)$ & 0.899 \\
\hline$<=5$ & Ref & & \\
\hline$>5$ & $2 \cdot 89$ & $(1 \cdot 38,6 \cdot 08)$ & 0.005 \\
\hline
\end{tabular}

TPPA, Treponema pallidum particle agglutination assay; TRUST, Toluidine red unheated serum test; Cerebrospinal fluid, CSF; Immunoglobulin, IgG. *Data with normal distribution was described using mean (sd).

\section{Laboratory findings and diagnostic yield}

The univariable logistic regression analysis revealed significant differences in psychiatric symptoms between the reactive NS group and control group (odds ratio [OR], 3·89; $95 \%$ confidence interval [CI], 1·70-8·89, $\mathrm{P}=0 \cdot 0001$; Table 2 ). The serum TRUST titres above 1:8 showed associations with NS diagnosis. However, no difference in creatine kinase levels was observed between the confirmed reactive NS and control groups (OR, 1.0; 95\% $\mathrm{Cl}, 1 \cdot 00-1 \cdot 01)$. Moreover, there were no significant differences between these two groups in CSF protein $>0 \cdot 45 \mathrm{~g} / \mathrm{L}(\mathrm{OR}, 4 \cdot 80 ; 95 \% \mathrm{Cl}, 2 \cdot 22-10 \cdot 36, \mathrm{P}<0.0001)$ and $\mathrm{CSF}$ nucleated cells $>5$ cells/L (OR, 2.89; 95\% Cl, 1·38-6.08, $\mathrm{P}=0 \cdot 005)$.

Table 3

\section{Multivariable logistic analysis for the construction of diagnostic models}




\begin{tabular}{|c|c|c|c|c|}
\hline & \multicolumn{3}{|c|}{ Development Cohort } & \multirow{2}{*}{$\begin{array}{l}\text { Validation Cohort } \\
\text { P }\end{array}$} \\
\hline & Multivariate & $\mathrm{P}$ & Multivariate & \\
\hline Male & $1 \cdot 0(0 \cdot 3,3 \cdot 1)$ & 0.954 & $1 \cdot 5(0 \cdot 5,4 \cdot 5)$ & $0 \cdot 43$ \\
\hline Psychiatric symptoms & $5 \cdot 1(1 \cdot 7,15 \cdot 4)$ & 0.004 & $1 \cdot 4(0 \cdot 4,4 \cdot 5)$ & 0.57 \\
\hline \multicolumn{5}{|l|}{ Serum TRUST } \\
\hline Negative & Ref & & Ref & \\
\hline 1 & $3 \cdot 2(0 \cdot 6,18 \cdot 8)$ & $0 \cdot 194$ & $8 \cdot 7(1 \cdot 0,75 \cdot 0)$ & 0.05 \\
\hline 8 & $7 \cdot 9(1 \cdot 1,54 \cdot 5)$ & 0.036 & $18 \cdot 8(1 \cdot 7,209 \cdot 6)$ & 0.02 \\
\hline 16 & $16 \cdot 0(2 \cdot 6,97 \cdot 7)$ & 0.003 & $45 \cdot 0(2 \cdot 2,937 \cdot 4)$ & 0.01 \\
\hline 32 & $33 \cdot 6(5 \cdot 4,209 \cdot 7)$ & $<0.001$ & $\inf \cdot(0 \cdot 0, \operatorname{lnf})$ & 0.99 \\
\hline 64 & $71 \cdot 9(9 \cdot 1,570 \cdot 0)$ & $<0.001$ & $60 \cdot 0(3 \cdot 0,1185.1)$ & 0.01 \\
\hline
\end{tabular}

TRUST, Toluidine red unheated serum test; Immunoglobulin, IgG; Cerebrospinal fluid, CSF Multivariable logistic analysis indicted in Table 3 that the following factors were more likely related to confirmed NS: serum TRUST 1:8 (OR, 7.9; 95\% Cl, 1·1-54.5), 1:16 (OR, 16·0; 95\% Cl,]·6-97·7), 1:32 (OR, 33.6; 95\% Cl, 5·4209.7), and 1:64 (OR, 71.9; $95 \% \mathrm{Cl}, 9 \cdot 1-570 \cdot 0)$, psychiatric symptoms (OR, 5.1; $95 \% \mathrm{Cl}, 1 \cdot 7-15 \cdot 4)$ and male $(0 \mathrm{R}, 1 \cdot 00 ; 95 \% \mathrm{Cl}, 0 \cdot 3-3 \cdot 1)$.

Table 4

Accuracy of the diagnostic score of the nomogram for estimating the risk of reactive neurosyphilis

\begin{tabular}{|lll|}
\hline & Value & \\
\hline Variable & Development Cohort & Validation Cohort \\
\hline ROC area (AUC) 95\% Cl & $0.85(0 \cdot 77,0 \cdot 91)$ & $0 \cdot 85(0 \cdot 74,0.93)$ \\
\hline Specificity, \% & 80.0 & $77 \cdot 5$ \\
\hline Sensitivity, \% & 79.0 & $81 \cdot 5$ \\
\hline Positive predictive value, \% & 90.8 & $71 \cdot 0$ \\
\hline Negative predictive value, \% & 60.3 & 86.1 \\
\hline
\end{tabular}

Receiver operating characteristics (ROC) curve of the model was generated using gender, psychiatric symptoms, and serum TRUST for neurosyphilis diagnosis. Diagnose-odds ratio (OR), positive predictive value (pv), and negative pv were calculated. *Area Under Curve (AUC) confidence interval and significance test adopted 500-time bootstrap resampling

The sensitivity and specificity of the diagnostic model in the development cohort were $80 \cdot 0$ and $79 \cdot 0$, respectively, and those in the validation cohort were $77 \cdot 5$ and $81 \cdot 5$, respectively (Table 4). The receiver operating characteristics (ROC) curve of the model was similar in the development (0.85, 95\% Cl: $0 \cdot 77-0 \cdot 91)$ and validation cohorts $(0 \cdot 85,95 \% \mathrm{Cl}: 0 \cdot 74-0 \cdot 93)$.

Figure 1 illustrates the nomogram with multi-variables for the diagnostic probability of reactive NS (Figure, A). The nomogram demonstrated good accuracy in estimating the diagnostic probability of reactive NS, with a bootstrap-corrected C index of 0.83 (Figure, B). The calibration plots were graphically good in the validation cohort; the nomogram displayed a $\mathrm{C}$ index of 0.71 for the estimation of reactive NS diagnosis (Figure, C).

\section{Discussion}

In this study, two important clinical findings were achieved. First, sex, psychiatric behaviour disorders, and serum TRUST titre were found to be useful indicators for the diagnosis of NS without lumbar puncture. Second, a diagnostic model for NS was developed and validated using clinical characteristics and laboratory test data of patients and transformed into a nomogram.

High serum TRUST titres are common in patients with syphilis. However, in this study, we found that serum the TRUST titre in conjugation with sex and psychiatric behaviour disorders could be used to differentiate patients with reactive NS from those suspected of having NS. Comparing the results with those of non-reactive NS and precluded patients, the serum TRUST levels were higher in patients with reactive NS. Cai et al. also indicated a 5-fold increased likelihood of asymptomatic NS in patients with a serum TRUST titre $\geq 1: 64 .{ }^{17}$ Researchers noted that an increase in the serum TPPA titre and serum creatine kinase could serve as a surrogate for CSF clinical abnormalities after lumbar punctures. ${ }^{17,18}$ Unfortunately, we were unable to determine the titre grades of serum TPPAs, since the laboratory system of our hospital automatically sets and reports serum TPPA titres $>1: 320$ as positive. Xiao et al. suggested that elevated serum creatine kinase may indicate NS among HIV-negative patients with syphilis. ${ }^{18}$ However, our data showed no significant difference in serum creatine kinase among reactive NS, non-reactive NS, and non-NS patients.

We used an exploratory approach combing clinical parameters and serum TRUST titres to develop diagnostic models for NS. When combined with clinical parameters, the diagnostic performance improved compared to that with the use of serum TRUST alone. The number of male patients was three times higher than that of female patients in our cohort, and is similar to that reported by the Public Health England. ${ }^{19}$ The rate of the presence of classic symptoms and

Page $7 / 10$ 
photophobia found in this study is consistent with that reported by Arielle, and is approximately $10 \%$ in HIV-negative patients with NS. ${ }^{9}$ The rate of NS typical symptoms like Argyll Robertson pupils was seldom reported. Instead, the significant clinical parameters observed in this study were psychiatric symptoms and memory deterioration, consistent with the Canadian and European case series reports of NS. ${ }^{20,21}$

An ROC curve analysis was used to assess the diagnostic model's performance. Approximately $85 \%$ of the AUC of the diagnostic model was similar in the development and validation cohorts. When we consider that lumbar puncture may be difficult for patients, nomogram having about $80 \%$ diagnostic probability may be sufficient to diagnose a patient with NS. However, in most cases, an $80 \%$ threshold probability is not sufficient, especially in cases with a high threshold to perform continuous intravenous antibacterial therapy. In such cases, it is better to complete CSF tests and conduct more specific diagnostic tests, such as the Venereal Disease Research Laboratory (VDRL) on the CSF, a well-known specific test, broadly used to diagnose idiopathic NS in America, although it is time-consuming and less feasible, especially in countries with high rates of such patients. ${ }^{22}$ The results of this study offer a sensitive screening nomogram for insisting candidates with high diagnostic possibility of reactive NS to undergo lumbar puncture, complete CSF regular tests, or undergo CSFVDRL measurements. Furthermore, this nomogram was cheaper than a regular procedure in terms of prices of medical services and tests, referring to prices of these items in 2021 from West China hospital (Supplement 2).

This study had several limitations. First, the study might have a sampling bias. We did not exclude patients who received insufficient antibiotic therapy before lumbar puncture. Under the current criterion of group assignment, false negatives were possible due to non-reactive NS cases. In theory, disease duration should have been analysed as a risk factor for NS, but it is difficult for patients with neuropsychological symptoms to provide the exact time of syphilis infection or information on sexual activities. Additionally, another limitation was the small sample size and that the pathological categories of NS with sophisticated infectious degree and loci were not employed here. Whether patients in each dedicated category had a different prognosis remains unknown due to lack of follow-up investigation. We intend to build a systematic database and prospectively design new studies to improve the quality of the evidence and facilitate more comprehensive patient care. In conclusion, to verify the validity of this model, future studies are warranted.

\section{Conclusion}

Importantly, an economical nomogram can be offered to assist clinicians and primary health servers in updating patients' medical charts and making informed decisions on NS diagnosis.

\section{Abbreviations}

CSF: cerebrospinal fluid

NS: neurosyphilis

TP: Treponema pallidum

TPCA: Treponema pallidum chemiluminescence assay

TPPA: Treponema pallidum particle agglutination assay

TRUST: toluidine red unheated serum test

VDRL: Venereal Disease Research Laboratory

\section{Declarations}

\section{Ethics approval and consent to participate}

All methods were carried out in accordance with Transparent Reporting of a multivariable prediction model for Individual Prognosis or Diagnosis (TRIPOD) guidelines and regulations.

Ethics committee on biomedical research, West China Hospital of Sichuan University approved the study and waived informed consent from all subjects. The committee's reference number was 1163 in 2020 approval.

\section{Consent for publication}

Not applicable

\section{Availability of data and materials}

The datasets used and/or analysed during the current study available from the corresponding author on reasonable request.

\section{Competing interests}


The authors declare that they have no competing interests

\section{Funding}

This work was funded by the National Key Research and Development Program of China (Nos. 2018YFC1311400, 2018YFC1311401) and Clinical Research Incubation Project of West China Hospital of Sichuan University (Grant 2018HXFH022)

\section{Authors' contributions}

WJ G analysed and interpreted the patient data regarding neurosyphilis and was a major contributor in writing the manuscript. YZ and LH instructed the study design of clinical research. CP performed part of lumber puncture. DD L instructed the interpretation of laboratory tests. LJ G instructed the data processing. JJ B and CL L helped process ethics profile. NC and LH provided the support of project grants. DZ proposed the study direction of neurosyphilis. All authors read and approved the final manuscript.

\section{Acknowledgements}

Not applicable

\section{References}

1. Ropper AH. Neurosyphilis. N Engl J Med. 2019 Oct 3;381(14):1358-1363. doi: 10.1056/NEJMra1906228. Erratum in: N Engl J Med. 2019 Oct 31;381(18):1789. PMID: 31577877.

2. Dombrowski JC, Pedersen R, Marra CM, Kerani RP, Golden MR. Prevalence Estimates of Complicated Syphilis. Sex Transm Dis. 2015 Dec;42(12):702-4. doi: $10.1097 /$ OLQ

3. CDC Sexually Transmitted Diseases Surveillance. 2015. http://www.cdc.gov/std/stats15/sysphilis.http

4. Tang W, Huang S, Chen L, Yang L, Tucker JD, et al. Late Neurosyphilis and Tertiary Syphilis in Guangdong Province, China: Results from a Cross-sectional Study. Rep, 2017, 7:45339.

5. Zhu L, Gu X, Peng RR, Wang C, Gao Z, et al. Comparison of the cerebrospinal fluid (CSF) toluidine red unheated serum test and the CSF rapid plasma reagin test with the CSF venereal disease research laboratory test for diagnosis of neurosyphilis among HIV-negative syphilis patients in China. $\mathrm{J}$ Clin Microbiol. 2014 Mar;52(3):736-40. doi: 10.1128/JCM.02522-13.

6. Clement ME, Okeke NL, Hicks CB. Treatment of syphilis: a systematic review. JAMA. 2014 Nov 12;312(18):1905-17. doi: 10.1001/jama.2014.13259.

7. Dumaresq J, Langevin S, Gagnon S, Serhir B, Deligne B, et al. Clinical prediction and diagnosis of neurosyphilis in HIV-infected patients with early Syphilis. Journal of Clinical Microbiology, 2013, 51(12):4060-4066.

8. Marra CM, Maxwell CL, Smith SL, Lukehart SA and Barnett SH. Cerebrospinal Fluid Abnormalities in Patients with Syphilis: Association with Clinical and Laboratory Features. The Journal of Infectious Diseases, 2004, 189(3):369-376.

9. Zhang HL, Lin LR, Liu GL, Zeng YL, Wu JY, et al. Clinical Spectrum of Neurosyphilis among HIV-Negative Patients in the Modern Era[J]. Dermatology, 2013, 226(2):148-156.

10. Davis AP, Stern J, Tantalo L, Sahi S, Holte S, et al. How Well Do Neurologic Symptoms Identify Individuals With Neurosyphilis? Clin Infect Dis. 2018 Jan 18;66(3):363-367. doi: 10.1093/cid/cix799

11. Choe PG, Song JS, Song KH, Jeon JH, Park WB, et al. Usefulness of routine lumbar puncture in non-HIV patients with latent syphilis of unknown duration[J]. Sexually Transmitted Infections, 2010.

12. Workowski KA, Bolan GA. Centers for Disease Control and Prevention. Sexually transmitted diseases treatment guidelines, 2015. MMWR Recomm Rep. 2015 Jun 5;64(RR-03):1-137.Janier M, Hegyi V, Dupin N, Unemo M, Tiplica GS, et al. 2014 european guideline on the management of syphilis. Journal of the European Academy of Dermatology \& Venereology, 2014 28(12), 1581-93.

13. Tuddenham S, Ghanem KG. Neurosyphilis: Knowledge Gaps and Controversies. Sex Transm Dis, 2018 March; 45(3):147-151.

14. Kingston M, French P, Higgins S, McQuillan O, Sukthankar A, et al. Members of the Syphilis guidelines revision group 2015, Radcliffe K, Cousins D, FitzGerald M, Fisher M, Grover D, Higgins S, Kingston M, Rayment M, Sullivan A. UK national guidelines on the management of syphilis 2015. Int J STD AIDS. 2016 May;27(6):421 - 46. doi: 10.1177/0956462415624059

15. Chinese Sexual Transmitted Disease Center. Guidelines for diagnosis and treatment of syphilis, gonorrhea, genital herpes, genital tract Chlamydia trachomatis infection, Chinese Journal of Dermatology, 2014, 47(5). doi: 10.3760/cma.j.issn.0412-4030.2014.05.022

16. Xiao Y, Tong ML, Liu LL, Lin, LR, Chen MJ, et al. Novel predictors of neurosyphilis among HIV-negative syphilis patients with neurological symptoms: an observational study[J]. BMC Infectious Diseases, 2017, 17(1):310.

17. Dumaresq J, Langevin S, Gagnon S, Tremblay C, et al. 2013. Clinical prediction and diagnosis of neurosyphilis in hiv-infected patients with early syphilis. Journal of Clinical Microbiology, 51(12), 4060-4066.

18. Xiao Y, Tong ML, Liu LL, Lin, LR, Chen MJ, et al. Novel predictors of neurosyphilis among HIV-negative syphilis patients with neurological symptoms: an observational study[J]. BMC Infectious Diseases, 2017, 17(1):310.

Page $9 / 10$ 
19. Public Health England. Addressing the increase in syphilis in England: Phe action plan, 2019. Available:

https://assets.publishing.service.gov.uk/government/uploads/system/uploads/attachment_data/file/806076/Addressing_the_increase_in_syphilis_in_En! Accessed 24 Jun 2019.

20. Marra CM, Tantalo LC, Maxwell CL, Ho EL, Sahi SK, Jones T. The rapid plasma reagin test cannot replace the venereal disease research laboratory test for neurosyphilis diagnosis. Sex Transm Dis. 2012;39(6):453-457. doi: 10.1097/OLQ.0b013e31824b1cde

21. Beauchemin P, Laforce R. Neurocognitive changes in tertiary neurosyphilis: a retrospective chart review.[J]. Canadian Journal of Neurological Sciences Le Journal Canadien Des Sciences Neurologiques, 2014, 41(04):452-458.

22. Ouwens ID, Koedijk F, Fiolet A, et al. Neurosyphilis in the mixed urban-rural community of the Netherlands[J]. Acta Neuropsychiatrica, 2014, 26(03):186192.

\section{Figures}

\section{A. Nomograom}

Points

Serum TRUST

Male

Psychiatric symptoms

Total Points

Linear Predictor

Risk of Event

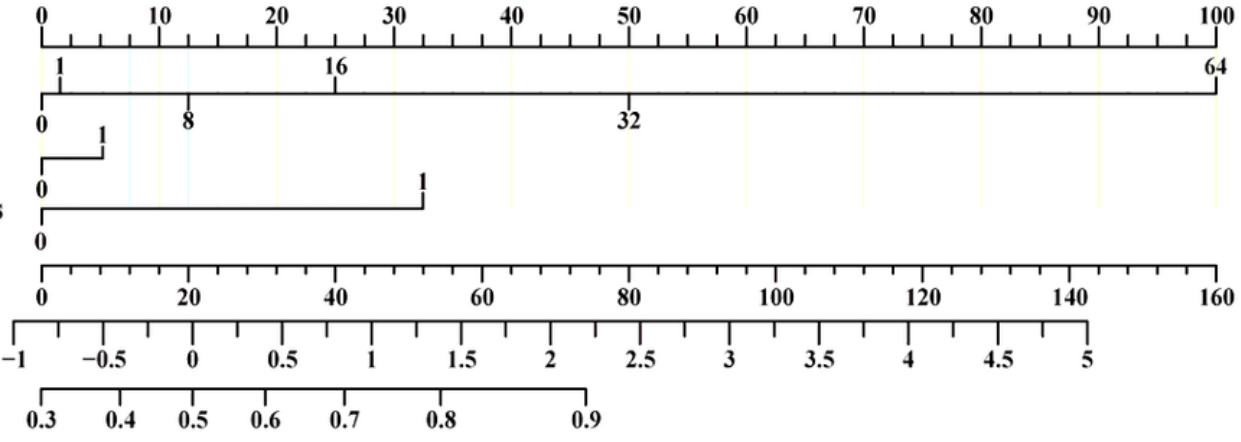

\section{B. Development Cohort}

B. Validation Cohort
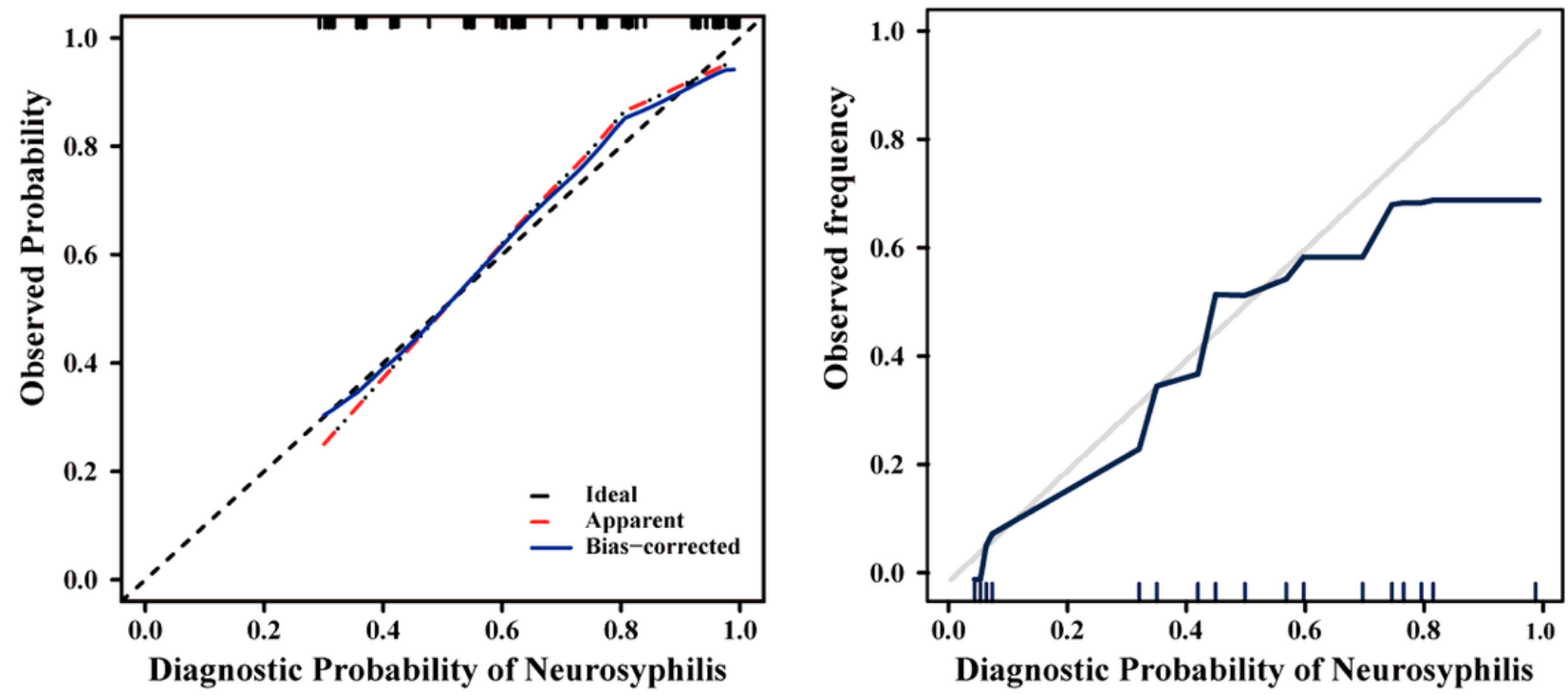

Figure 1

ROC curve, DCA, and nomograms(a) (A) Nomogram visualising the multivariable diagnostic model. To use the nomogram, find the position of each variable on the corresponding axis, draw a line to the points axis for the number of points, add the points from all variables, and draw a line from the total points axis to assess diagnostic probability of NS at the lower line of the nomogram. (B) Calibration curves of the nomogram for the diagnostic probability of NS in the training cohort $(n=148)$. (C) Validity of the performance of the nomogram in estimating the diagnostic probability of NS in the validation cohort $(n=67)$.

*AUC confidence interval and significance test adopt Bootstrap resampling 500 times

\section{Supplementary Files}

This is a list of supplementary files associated with this preprint. Click to download.

- Supplementtables.docx 